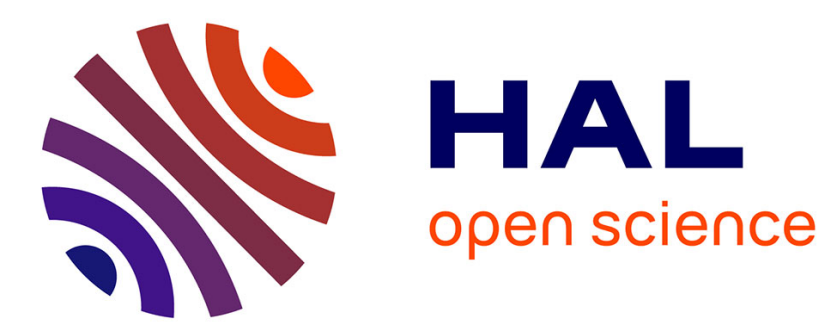

\title{
On the Internal and External Stability of Coalitions and Application to Group Purchasing Organizations
}

\author{
Dongshuang Hou, Aymeric Lardon, Hao Sun
}

\section{To cite this version:}

Dongshuang Hou, Aymeric Lardon, Hao Sun. On the Internal and External Stability of Coalitions and Application to Group Purchasing Organizations. 2020. halshs-02860639

\section{HAL Id: halshs-02860639 \\ https://shs.hal.science/halshs-02860639}

Preprint submitted on 8 Jun 2020

HAL is a multi-disciplinary open access archive for the deposit and dissemination of scientific research documents, whether they are published or not. The documents may come from teaching and research institutions in France or abroad, or from public or private research centers.
L'archive ouverte pluridisciplinaire HAL, est destinée au dépôt et à la diffusion de documents scientifiques de niveau recherche, publiés ou non, émanant des établissements d'enseignement et de recherche français ou étrangers, des laboratoires publics ou privés. 
UMR 5824

93. chemin des Mouilles 69130 Ecully - France

Maison de l'Universite, Bâtiment B 10, rue Trefilerie 42023 Saint-Etienne cedex $02 \cdot$ France http://www.gate.cnrs.fr gate@gate.cnrs.fr

\title{
On the Internal and External Stability of Coalitions and Application to Group Purchasing Organizations
}

\author{
Dongshuang Hou, Aymeric Lardon, Hao Sun
}

\begin{abstract}
:
Two new notions of stability of coalitions, based on the idea of exclusion or integration of players depending on how they affect allocations, are introduced for cooperative transferable utility games. The first one, called internal stability, requires that no coalition member would find that her departure from the coalition would improve her allocation or those of all her partners. The second one, called external stability, requires that coalitions members do not wish to recruit a new partner willing to join the coalition, since her arrival would hurt some of them. As an application of these two notions, we study the stability of Group Purchasing Organizations using the Shapley value to allocate costs between buyers. Our main results suggest that, when all buyers are initially alone, while small buyers will form internally and externally stable Group Purchasing Organizations to benefit from the best price discount, big buyers will be mutually exclusive and may cooperate with only small buyers.
\end{abstract}

\section{Keywords:}

Internal and external stability, Group purchasing organization, Cost allocation, Shapley value

\section{JEL codes:}

C71, D61, D62 


\title{
On the Internal and External Stability of Coalitions and Application to Group Purchasing Organizations
}

\author{
DONGSHUANG HOU * \\ AYMERIC LARDON ${ }^{\dagger}$ \\ HAO SUN $\ddagger$
}

June 2, 2020

\begin{abstract}
Two new notions of stability of coalitions, based on the idea of exclusion or integration of players depending on how they affect allocations, are introduced for cooperative transferable utility games. The first one, called internal stability, requires that no coalition member would find that her departure from the coalition would improve her allocation or those of all her partners. The second one, called external stability, requires that coalitions members do not wish to recruit a new partner willing to join the coalition, since her arrival would hurt some of them. As an application of these two notions, we study the stability of Group Purchasing Organizations using the Shapley value to allocate costs between buyers. Our main results suggest that, when all buyers are initially alone, while small buyers will form internally and externally stable Group Purchasing Organizations to benefit from the best price discount, big buyers will be mutually exclusive and may cooperate with only small buyers.
\end{abstract}

Keywords: Internal and external stability; Group purchasing organization; Cost allocation; Shapley value

JEL Classification System: C71; D61; D62

\section{Introduction}

Cooperative game theory aims to study situations where groups of players, called coalitions, can coordinate their actions in order to attain better results than they could attain by acting individually. The game is said to be a transferable utility game (henceforth TU-game) if the worth of coalitions can be represented by a real number interpreted as money value, reflecting benefits or costs, which can be freely divided through side payments between coalition members. In the framework of TU-games, a wide variety of solutions have been proposed to allocate in

*Dongshuang Hou, Department of Applied Mathematics, Northwestern Polytechnical University. The first author acknowledges financial support by National Science Foundation of China (NSFC) through grant No. 71871180,71671140 .

†Aymeric Lardon, Univ Lyon, UJM Saint-Etienne, CNRS, GATE Lyon Saint-Etienne UMR 5824, F-42023 Saint-Etienne, France. Author in charge of correspondence. E-mail: aymeric.lardon@univ-st-etienne.fr

${ }^{\ddagger}$ Hao Sun, Department of Applied Mathematics, Northwestern Polytechnical University. 
a fair way the worth of the grand coalition (i.e. the whole set of players) to each player. On the one hand, some solutions, called allocation rules or values, are defined from a set of criteria of distributive justice. The most prominent example of this solution type is undoubtly the Shapley value (Shapley, 1953 [14]) that gives to each player her average marginal contribution to the worth of all possible coalitions. The Shapley value has been largely studied in literature by, among others, Young (1985 [18]), Chun (1991 [2]) and Hou et al. (2018 [6]). On the other hand, another solution type solves allocation problems by considering the blocking power of coalitions which represents their abilities to negotiate a "slice of the pie" during the sharing process. The core (Shapley, 1955 [15]) of TU-games is based on such no-blocking conditions by selecting allocations from which no coalition is dissatisfied about the payoffs or costs distributed to its members. Thus, solutions in TU-games can be regarded as tools to assess the legitimacy of allocations selected by regulator seeking a compromise between players.

In this article, we propose to analyze the stability of coalitions in TU-games from a new angle. Precisely, we assume that players have already agreed on the value to use to allocate the worth of the grand coalition. ${ }^{1}$ Once a value is selected, coalition members can then contest whether or not a player can belong to the coalition depending on how she affects allocations. This leads us to consider two stability types. The first one states that a coalition is "internally stable" if there exists no coalition member who would find that her departure from the coalition would improve her allocation or those of all her partners. In this latter case, the other members would unanimously decide to exclude this harmful player. The second one states that a coalition is "externally stable" if there is no incentive for coalition members to recruit a new partner willing to join the coalition, since her arrival would hurt some of them. In that case, coalition members would decide to keep out this undesirable player. To the best of our knowledge, we are not aware of internal and external stability notions of coalitions, based on values in TU-games. A few decades ago, these notions have been considered to define stable sets by von Neumann and Morgenstern (1944 [10]). They have also been formalized by d'Aspremont et al. (1983 [3]) and used by Thoron (1998 [16]) in the study of cartel formation.

The notions of internal and external stability of coalitions are relevant in a wide variety of situations such as, among many others, purchasing, transportation, natural resources and cartels. In this article, we propose to study the stability of Group Purchasing Organizations (henceforth GPOs) which are formed in various economic sectors such as, for example, health care, education and retail. GPOs are purchasing coalitions of several buyers (for example hospitals, schools or states) that realize savings and efficiencies by aggregating purchasing volume and using that leverage to negotiate discounts with sellers. Recently, GPOs are a key part of the overall effort to fight the COVID-19 pandemic as Khatereh Calleja, CEO of Healthcare Supply Chain Association, said in a statement: "As the United States continues to address the spread of COVID-19, supporting healthcare providers and the patients they serve is more important than ever. GPOs are on the front lines of patient care, working with provider partners to deliver a safe and reliable supply of the best products at the best value." Despite the various benefits of forming GPOs (Doucette, 1997 [5]; Zentes and Swoboda, 2000 [19], Safaei et al., 2017 [11]), academic literature brings to light that these organizations often disappear by themselves, in part because of disagreements about cost allocations between their members (Schotanus et al., 2008 [13]). Furthermore, Bloch et al. (2008, [1]) observe that the membership numbers of GPOs often fluctuate due to both unfair cost allocation rules and unequal member contributions. As a consequence, some coalition members may decide to exclude some of their partners or build

\footnotetext{
${ }^{1}$ Equivalently, the selected value can also be imposed on players by regulator.
} 
barriers to keep out new participants from entering GPO.

Although it has been shown that GPOs have considerable power to influence price market (Yang et al., 2017 [17]) and increase the profits of their members (Li, 2019 [7]), they received relatively little attention in theoretical economics and operational research to offer any robust remedy against their unstability in the long run. To face these new challenges, we consider the framework of cost TU-games to solve cost allocation problems within GPOs. Precisely, we assume that a set of buyers, each one with a certain purchasing requirement of the same good, decide to form a purchasing coalition. Furthermore, the price discount proposed by seller depends on the aggregated purchasing quantity. Thus, each coalition member is able to receive a lower price than the one she would have received based solely on her individual order. The functional form we use to represent price discount schedules fits best with what is seen in practice (Schotanus, 2007 [12]), and has several interesting analytical properties as well. ${ }^{2}$ Our analysis proceeds along the following lines. For any GPO, we first compute the Shapley value of the associated cost TU-game, called GPO game, in order to provide fair cost allocation recommendations (Propositions 3.1, 3.4 and 3.11). Then, using the Shapley value of GPO games, we study the internal and external stabilty of GPOs by comparing the cost each buyer has to pay before and after the departure or arrival of any participant. On the one hand, if a buyer left an internally stable GPO, her departure would increase her cost and, either increase the cost of at least one of her partners or left unchanged their costs. On the other hand, with an externally stable GPO there is no incentive for coalition members to recruit a new buyer willing to join the GPO, since her arrival would either increase the cost of at least one of them or, at most, left unchanged their costs. To facilitate the analysis, we distinguish small and big buyers within GPOs. While a small buyer cannot attain the best price discount by herself, a big buyer is willing to buy sufficient quantities of goods to obtain such a price.

First, we show that GPOs with only small buyers have interest to recruit new small ones to reduce the costs of their members as long as the best price discount is not attained (Proposition 3.2). As a consequence, such GPOs are internally stable but not externally stable (Corollary 3.3). Furthermore, when all small buyers of GPO are necessary to attain the best price discount, we provide sufficient conditions under which GPO has still interest to recruit new small buyers (Proposition 3.5). Hence, such GPOs may also be internally stable but not externally stable (Corollaries 3.6 and 3.7). Second, we establish that the GPO comprising all small buyers, and only them, is both internally and externally stable under general conditions on price discount schedules and demanded quantities (Proposition 3.9 and Theorem 3.10). When only big buyers exist, we also prove that any GPO is internally and externally stable (Corollary 3.13). Finally, for GPOs comprising both small and big buyers, we show that big buyers are mutually exclusive and cannot coexist within GPO (Proposition 3.14). Hence, such GPOs are externally stable when they already comprise all small buyers (Corollary 3.15). In summary, our main results suggest that, when all buyers are initially alone, while small buyers will form internally and externally stable GPOs to benefit from the best price discount, big buyers will be mutually exclusive and may cooperate with only small buyers (Corollary 3.17).

The rest of the article is organized as follows. In Section 2, we introduce the framework of cost TU-games as well as the notions of internal and external stability of coalitions. In Section 3, we determine the Shapley value of GPO games and we study the stability of GPOs. Section 4 concludes. All proofs appear in the Appendix.

\footnotetext{
${ }^{2}$ Schotanus (2007 [12]) analyzes price discount schedules that are often used in GPOs and exploits these data to fit a function that best describes the contracts in horizontal cooperative purchasing.
} 


\section{Preliminaries}

\subsection{Cost TU-games and solutions}

Let $\mathcal{U}$ be a nonempty set of players. The set $\mathcal{U}$ may be finite or infinite. A nonempty and finite subset $N \subseteq \mathcal{U}$ is called a coalition, whose cardinality will be denoted by $n$. A cost TU-game is a pair $(N, c)$ where $N$ is a coalition and $c: 2^{N} \longrightarrow \mathbb{R}$ is a cost function such that $c(\emptyset)=0$. For any coalition $S \subseteq N, c(S)$ represents the least cost of serving the members of $S$ by the most efficient means. The set of cost TU-games is denoted by $\Gamma$. A cost TU-game $(N, c)$ is subadditive if for all $S, T \subseteq N$ with $S \cap T=\emptyset, c(S)+c(T) \geq c(S \cup T)$, and $(N, c)$ is concave if for all $S, T \subseteq N, c(S)+c(T) \geq c(S \cup T)+c(S \cap T)$. A subgame of a cost TU-game $(N, c)$ is a game $\left(T, c^{T}\right) \in \Gamma$ where $\emptyset \neq T \subseteq N$ and $c^{T}(S)=c(S)$ for all $S \subseteq T$. For notational convenience, the subgame $\left(T, c^{T}\right)$ will also be denoted by $(T, c)$.

A fundamental question in cooperative game theory concerns how to allocate costs between players according to some criteria of distributive justice. A value is a mapping $\phi$ on $\Gamma$ which uniquely determines, for each $(N, c) \in \Gamma$, and each $i \in N$, a cost $\phi_{i}(N, c) \in \mathbb{R}$ player $i$ has to pay for participating in $(N, c)$. One of the most well-known values for cost TU-games is the Shapley value (Shapley, 1953 [14]) given by:

$$
S h_{i}(N, c)=\sum_{S \subseteq N \backslash\{i\}} \frac{s !(n-s-1) !}{n !}(c(S \cup\{i\})-c(S)), i \in N .
$$

The Shapley value has many appealing properties (Young, 1985 [18]; Chun, 1991 [2]; Hou et al., 2018 [6]) and has been successfully applied to a diversity of fields such as, among many others, voting, networks and environmental problems (see, for example, Moretti and Patrone, 2008 [8]). Another central question in cooperative game theory is how to allocate the total costs of the grand coalition between its members in such a way that no player or group of players can dispute cost allocations. This is basically a no-blocking condition underlying the core of cost TU-games (Shapley, 1955 [15]) given by:

$$
C(N, c)=\left\{x \in \mathbb{R}^{N}: \sum_{i \in N} x_{i}=c(N), \sum_{i \in S} x_{i} \leq c(S) \text { for all } S \subset N\right\} .
$$

It is widely known that the Shapley value is individually rational for subadditive cost TU-games, i.e. $S h_{i}(N, c) \leq c(\{i\})$ for all $i \in N$, and it belongs to the core for concave cost TU-games (Moulin, 1988 [9]). Thus, the lesson that can be drawn is that these two key concepts permit to assess the legitimacy of cost allocations between players selected by regulator.

\subsection{Internal and external stability of coalitions}

In this article, we propose to analyze the stability of coalitions from a new angle. Precisely, instead of testing the legitimacy ${ }^{3}$ of cost allocations, we assume that coalition members have already accepted cost allocation rules (that is, they already agreed on the value to use to allocate costs) and can then contest whether or not a player can belong to a coalition depending on how she affects cost allocations. To the best of our knowledge, we are not aware of any general

\footnotetext{
${ }^{3}$ Generally, this legitimacy either comes from some criteria of distributive justice used to design values, or is based on the non-blocking conditions of coalitions.
} 
stability concept of coalitions dealing with the exclusion or integration of players, based on a preselected value in cost TU-games. To formalize this idea, we distinguish between internal and external stability. A coalition $N \subseteq \mathcal{U}$ is internally stable with respect to $\phi$ if for all $j \in N$ :

$$
\begin{gathered}
\exists i \in N \backslash\{j\}, \phi_{i}(N, c)<\phi_{i}(N \backslash\{j\}, c), \\
\text { or } \\
\forall i \in N \backslash\{j\}, \phi_{i}(N, c) \leq \phi_{i}(N \backslash\{j\}, c), \\
\text { and } \\
\phi_{j}(N, c) \leq \phi_{j}(\{j\}, c) .
\end{gathered}
$$

If a coalition member left an internally stable coalition, it would find that her departure would increase her cost and, either increase the cost of at least one of her partners or left unchanged their costs. A coalition $N \subseteq \mathcal{U}$ is externally stable with respect to $\phi$ if for all $j \in \mathcal{U} \backslash N$ :

$$
\begin{gathered}
\exists i \in N, \phi_{i}(N \cup\{j\}, c)>\phi_{i}(N, c), \\
\text { or } \\
\forall i \in N, \phi_{i}(N \cup\{j\}, c) \geq \phi_{i}(N, c), \\
\text { or } \\
\phi_{j}(N \cup\{j\}, c)>\phi_{j}(\{j\}, c) .
\end{gathered}
$$

With an externally stable coalition there is no incentive for coalition members to accept a new partner willing to join them, since her arrival would either increase the cost of at least one of them or, at most, left unchanged their costs. ${ }^{4}$ In cooperative game theory, the notions of internal and external stability have first been considered to define stable sets by von Neumann and Morgenstern (1944 [10]). They have also been formalized by d'Aspremont et al. (1983 [3]) and used by Thoron (1998 [16]) in the study of cartel formation. These two notions, as defined above, are relevant to deal with the stability of coalitions in a wide variety of situations such as, among many others, purchasing, transportation, natural resources and cartels. To this end, the next section of this article is devoted to the study of the stability of GPOs.

\footnotetext{
${ }^{4}$ When the worth of coalitions reflects benefits instead of costs, it suffices to invert the inequalities to obtain the appropriate notions of internal and external stability in TU-games.
} 


\subsection{GPO games}

From now on, we assume that $\mathcal{U}$ is a finite set of buyers who can decide to form a Group Purchasing Organization $N \subset \mathcal{U}$ to combine their individual requirements for goods to gain better prices. ${ }^{5}$ The goods are homogeneous and the demand vector $q \in R_{++}^{N}$ is a $n$-dimensional vector assigning a demanded quantity $q_{i} \in \mathbb{R}_{++}$to each buyer $i$. For any coalition $S \subseteq N$, the quantity $Q(S)=\sum_{i \in S} q_{i}$ represents the total quantity of goods that the members of $S$ are willing to buy in order to meet their consumption needs. The price function $p: \mathbb{R}_{+} \longrightarrow \mathbb{R}_{+}$assigns a unit purchase price $p(Q)$ to each aggregated quantity $Q \in \mathbb{R}_{+}$and is given by:

$$
p(Q)= \begin{cases}a-b Q, & \text { if } Q<V \\ a_{\text {min }}, & \text { otherwise }\end{cases}
$$

where $\left(a, b, a_{\text {min }}, V\right) \in \mathbb{R}_{++}^{4}$ are such that $0<a_{\text {min }}<a-b V$. The real numbers $a, b, a_{\text {min }}$ and $V$ can be respectively interpreted as the original price, the discount rate, the best price discount and the threshold purchase quantity. Observe that the price function $p$ is piecewise linear and decreasing in $Q$ (so, it reflects a discount), and becomes constant when $Q$ is greater than or equal to $V$.

Given the triplet $(N, q, p)$, a GPO game is a cost TU-game $(N, c) \in \Gamma$ where $N$ is a set of buyers forming a GPO and $c: 2^{N} \longrightarrow \mathbb{R}$ is a cost function given by:

$$
c(S)=p(Q(S)) Q(S)= \begin{cases}(a-b Q(S)) Q(S), & \text { if } Q(S)<V \\ a_{\min } Q(S), & \text { otherwise }\end{cases}
$$

On the one hand, if the total purchases $Q(S)$ of coalition $S$ are less than the threshold purchase quantity $V$, then the unit purchase price is decreasing with respect to $Q(S)$. On the other hand, if the the total purchases $Q(S)$ of coalition $S$ are greater than or equal to $V$, then the unit purchase price drops to the lowest level $a_{\text {min }}$. Thus, while there are incentives for GPO members to include new buyers in order to get better prices, the marginal benefits tend to be smaller as GPO becomes larger which may lead GPO members to exclude some of their partners. These two antagonist effects suggest that there would be an optimal size of GPO which can be analyzed with the notions of internal and external stability of coalitions previously defined. Formally, this means that GPO games are subadditive, since the price function is decreasing in $Q$, but not concave as illustrated by the following example.

Example 2.1. Let $(\{1,2,3\}, c) \in \Gamma$ be a GPO game such that the original price $a=10$, the discount rate $b=0.03$, the best price discount $a_{\text {min }}=6$, the threshold purchase quantity $V=100$, and the demand vector $\left(q_{1}, q_{2}, q_{3}\right)=(40,80,80)$. The worth of coalitions are given in the following table:

\begin{tabular}{|c|ccccccc|}
\hline$S$ & $\{1\}$ & $\{2\}$ & $\{3\}$ & $\{1,2\}$ & $\{1,3\}$ & $\{2,3\}$ & $\{1,2,3\}$ \\
\hline$c(S)$ & 352 & 608 & 608 & 720 & 720 & 960 & 1200 \\
\hline
\end{tabular}

Thus, we observe that $(\{1,2,3\}, c)$ is subadditive but not concave since $c(\{1,2\})+c(\{2,3\})=$ $1680<1808=c(\{1,2,3\})+c(\{2\})$.

\footnotetext{
${ }^{5}$ In practice, there are many other advantages in forming a GPO such as better design of goods, supply availability and assurance benefits. In our model, we focus exclusively on the price discount effects of GPOs.
} 


\section{Internal and External Stability of GPOs}

In this section, we assume that buyers are aware of and agree with the marginalist principle, of old tradition in economic theory, underlying the Shapley value. In words, buyers accept to allocate costs according to their marginal contributions to coalitions, that is, the additional cost they bring in. Furthermore, buyers aim to minimize their own expenses (costs) in order to satisfy their demands by questioning whether or not any buyer can belong to GPO depending on how she affects cost allocations. Thus, the notions of internal and external stability are relevant to study GPO formation. They first require to determine the Shapley value of GPO games. To this end, the set of buyers $\mathcal{U}$ is partitioned into the two subsets of small buyers $M=\left\{i \in \mathcal{U}: q_{i}<V\right\}$ and big buyers $\mathcal{U} \backslash M=\left\{i \in \mathcal{U}: q_{i} \geq V\right\}$. Moreover, since GPO games are subadditive, the Shapley value is individually rational, and any buyer is then always willing to join and stay within GPO. So, we only need to focus on the acceptance or not of buyers by other GPO members when dealing with the internal and external stability property. To facilitate the analysis, we first focus on GPOs comprising only small buyers. Then, we consider general GPOs integrating both small and big buyers.

\subsection{GPOs with only small buyers}

We first compute the Shapley value of GPO games when the total puchases of small buyers are less than the threshold purchase quantity $V$.

Proposition 3.1. For any GPO game $(N, c) \in \Gamma$ such that $Q(N)<V$, the Shapley value of $(N, c)$ is given by:

$$
S h_{i}(N, c)=q_{i}(a-b Q(N)), i \in N .
$$

It is worth noting that $Q(N)<V$ implies that $N \subseteq M$. In this case, Proposition 3.1 states that the cost buyer $i$ has to pay is a decreasing function of the total purchases $Q(N)$. The next result is a consequence of Proposition 3.1.

Proposition 3.2. For any GPO game $(N, c) \in \Gamma$ such that $Q(N)<V$, it holds that:

$$
S h_{i}(N, c)-S h_{i}(N \backslash\{j\}, c)=-b q_{i} q_{j}<0, i \in N, j \in N \backslash\{i\} .
$$

Thus, if a buyer $j \in N$ breaks off from coalition $N$, then the Shapley value of any buyer $i \in N \backslash\{j\}$ will increase. By a similar argument, we can say that if a small buyer $j \in M \backslash N$ such that $Q(N \cup\{j\})<V$ joins coalition $N$, then the Shapley value of any buyer $i \in N$ will decrease. Hence, we directly deduce the following result.

Corollary 3.3. For any GPO game $(N, c) \in \Gamma$ such that $Q(N)<V$, it holds that $N$ is internally stable. Moreover, if there exists $j \in M \backslash N$ such that $Q(N \cup\{j\})<V$, then $N$ is not externally stable.

This result states that when the total purchases of GPO do not exceed the threshold purchase quantity $V$, small buyers will always take advantage from the cooperation with their peers to reduce their expenses.

The next result determines the Shapley value of GPO games when all small buyers are necessary to get the best price discount $a_{\min }$. 
Proposition 3.4. For any GPO game $(N, c) \in \Gamma$ such that $N \subseteq M, Q(N \backslash\{i\})<V$ for all $i \in N$ and $Q(N) \geq V$, the Shapley value of $(N, c)$ is given by:

$$
S h_{i}(N, c)=q_{i}(a-b Q(N))-\frac{\left(a-b Q(N)-a_{m i n}\right) Q(N)}{n}, i \in N .
$$

In contrast to Proposition 3.1, the total purchases of the GPO satisfy $V \leq Q(N)<V+q_{i}$ for all $i \in N$. In this case, Proposition 3.4 states that the cost buyer $i$ has to pay is a decreasing function of the total purchases $Q(N)$ if and only if $a-a_{m i n}+b\left(n q_{i}-2 Q(N)\right) \geq 0$. The next result follows directly from Propositions 3.1 and 3.4.

Proposition 3.5. For any $\operatorname{GPO}$ game $(N, c) \in \Gamma$ such that $N \subseteq M, Q(N \backslash\{i\})<V$ for all $i \in N$ and $Q(N) \geq V$, it holds that:

$$
S h_{i}(N, c)-S h_{i}(N \backslash\{j\}, c)=-b q_{i} q_{j}-\frac{\left(a-b Q(N)-a_{\min }\right) Q(N)}{n}, i \in N, j \in N \backslash\{i\} .
$$

Thus, if $a-b Q(N)-a_{\text {min }} \geq 0$ then the cost allocated to any buyer $i \in N$ will increase when a buyer $j \in N \backslash\{i\}$ breaks off from coalition $N$. Analogously, the GPO members have interest to accept any small buyer $j \in M \backslash N$ when $Q((N \cup\{j\}) \backslash\{i\})<V$ for all $i \in N \cup\{j\}$, $Q(N \cup\{j\}) \geq V$, and $a-b Q(N \cup\{j\})-a_{\min } \geq 0$. Hence, we directly deduce the following two results.

Corollary 3.6. For any GPO game $(N, c) \in \Gamma$ such that $N \subseteq M, Q(N \backslash\{i\})<V$ for all $i \in N$, $Q(N) \geq V$ and $a-b Q(N)-a_{m i n} \geq 0$, it holds that $N$ is internally stable.

Corollary 3.7. For any GPO game $(N, c) \in \Gamma$ such that $N \subset M$, if there exists $j \in M \backslash N$ such that $Q((N \cup\{j\}) \backslash\{i\})<V$ for all $i \in N \cup\{j\}, Q(N \cup\{j\}) \geq V$ and $a-b Q(N \cup\{j\})-a_{\text {min }} \geq 0$, then $N$ is not externally stable.

Since $S h_{i}(N, c)-S h_{i}(N \backslash\{j\}, c) \geq 0$ may occur, Proposition 3.5 also reveals that some GPO members may seek to build barriers to keep out new buyers as illustrated by the following example.

Example 3.8. Let $\mathcal{U}=\{1,2,3\}$, and $(\{1,2\}, c),(\{1,2,3\}, c) \in \Gamma$ be two GPO games such that the original price $a=10$, the discount rate $b=0.003$, the best price discount $a_{\min }=6.5$, the threshold purchase quantity $V=1000$, and the demand vector $\left(q_{1}, q_{2}, q_{3}\right)=(200,800,800)$. Note that buyers 1, 2 and 3 are small buyers, $Q(N \backslash\{i\}) \geq V$ for all $i \in\{1,2,3\}$, and $a-b Q(N)-a_{\min }<$ 0 . Hence, the conditions of Propositions 3.4 and 3.5 are not satisfied. The worth of coalitions are given in the following table:

\begin{tabular}{|c|ccccccc|}
\hline$S$ & $\{1\}$ & $\{2\}$ & $\{3\}$ & $\{1,2\}$ & $\{1,3\}$ & $\{2,3\}$ & $\{1,2,3\}$ \\
\hline$c(S)$ & 1880 & 6080 & 6080 & 6500 & 6500 & 10400 & 11700 \\
\hline
\end{tabular}

Some calculations show that $S h_{1}(\{1,2\}, c)=1150, S h_{2}(\{1,2\}, c)=5350, S h_{1}(\{1,2,3\}, c)=$ 1200 , and $S h_{2}(\{1,2,3\}, c)=S h_{3}(\{1,2,3\}, c)=5250$. It is clear that coalition $\{1,2\}$ is internally stable. Moreover, the cost buyer 1 has to pay would increase by 50 if buyer 3 joins coalition $\{1,2\}$, proving that $\{1,2\}$ is also externally stable. 
So far, we have considered only small buyers to leave or join GPOs. The next result concerns the external stability of the GPO comprising all small buyers when big buyers may join it.

Proposition 3.9. For any GPO game $(N, c) \in \Gamma$ such that $N=M, Q(N \backslash\{i\})<V$ for all $i \in N$ and $Q(N) \geq V$, it holds that $N$ is externally stable.

When the GPO comprises all small buyers, and only them, Proposition 3.9 states that there exists at least one member who will reject the joining of any big buyer. Indeed, the unit purchase price of goods for coalition $N$ has already reached the best price discount $a_{\text {min }}$ since $Q(N) \geq V$. Furthermore, the marginal cost $a_{m i n} q_{j}$ of big buyer $j$ is greater than the cost $S h_{j}(N \cup\{j\}, c)$ she has to pay for participating in $(N \cup\{j\}, c)$ since the Shapley value is individually rational. Therefore, the cost of at least one small buyer within the GPO will increase after the arrival of big buyer $j$. Combining Corollary 3.6 and Proposition 3.9, we obtain the following result.

Theorem 3.10. For any GPO game $(N, c) \in \Gamma$ such that $N=M, Q(N \backslash\{i\})<V$ for all $i \in N$, $Q(N) \geq V$ and $a-b Q(N)-a_{m i n} \geq 0$, it holds that $N$ is both internally and externally stable.

Thus, the GPO comprising all small buyers, and only them, is internally and externally stable when all members are necessary to get the lowest price $a_{\text {min }}$, and the difference between the original price $a$ and the best price discount $a_{\text {min }}$ is sufficiently large in comparison with the total purchases $Q(N)$ weighted by the discount rate $b$.

\subsection{GPOs with small and big buyers}

We have seen in Proposition 3.9 that it is unlikely for GPOs with only small buyers to accept big buyers when their total purchases are greater than or equal to the threshold purchase quantity $V$. Thus, we will focus on situations where big buyers coexist with small buyers when the total purchases of these latter are less than $V$. The next result determines the Shapley value of GPO games with both small and big buyers when small buyers cannot reach the best price discount $a_{\text {min }}$.

Proposition 3.11. For any GPO game $(N, c) \in \Gamma$ such that $Q(T)<V$ where $T=N \cap M$, the Shapley value of $(N, c)$ is given by:

$$
S h_{i}(N, c)=\frac{n-t}{n-t+1} a_{\min } q_{i}+\frac{p(Q(T)) q_{i}}{n-t+1}+\frac{(n-t) b q_{i} Q(T \backslash\{i\})}{(n-t+1)(n-t+2)}, i \in T,
$$

and

$$
S h_{i}(N, c)=a_{\text {min }} q_{i}-\frac{\left(a-b Q(T)-a_{m i n}\right) Q(T)}{(n-t+1)(n-t)}-\frac{2 b \sum_{t, r \in T} q_{t} q_{r}}{(n-t+1)(n-t+2)}, i \in N \backslash T .
$$

It is important to note that Proposition 3.11 also determines the Shapley value of GPO games with only big buyers by considering $T=\emptyset$ in (3.4). Hence, we directly deduce the following result.

Corollary 3.12. For any GPO game $(N, c) \in \Gamma$ such that $N \cap M=\emptyset$, the Shapley value of $(N, c)$ is given by:

$$
S h_{i}(N, c)=a_{\min } q_{i}, i \in N
$$


Indeed, we can observe that any GPO game with only big buyers is additive ${ }^{6}$ which implies that the cost buyer $i$ has to pay is equal to her individual cost $c(\{i\})$. In this case, big buyers do not affect cost allocations within GPOs. Hence we obtain the following statement as a corollary to Proposition 3.11.

Corollary 3.13. For any GPO game $(N, c) \in \Gamma$, if $M=\emptyset$ then $N$ is both internally and externally stable.

The next result concerns GPOs with both small and big buyers and is a direct consequence of Proposition 3.11.

Proposition 3.14. For any GPO game $(N, c) \in \Gamma$ such that $Q(T)<V$ where $T=N \cap M$, if there exists $j \in \mathcal{U} \backslash(N \cup M)$, then:

$$
\begin{aligned}
S h_{i}(N \cup\{j\}, c)-S h_{i}(N, c) & =-\frac{\left(p(Q(T))-a_{\text {min }}\right) q_{i}}{(n-t+1)(n-t+2)} \\
& <0, i \in T, \quad-\frac{(n-t-1) b q_{i} Q(T \backslash\{i\})}{(n-t+1)(n-t+2)(n-t+3)}
\end{aligned}
$$

and

$$
\begin{aligned}
S h_{i}(N \cup\{j\}, c)-S h_{i}(N, c) & =\frac{2\left(a-b Q(T)-a_{\text {min }}\right) Q(T)}{(n-t)(n-t+1)(n-t+2)} \\
& >0, i \in N \backslash T .
\end{aligned}
$$

Thus, while the costs of small buyers in $T$ decrease, those of big buyers in $N \backslash T$ increase which implies that big buyer $j$ will not be accepted within the GPO. Hence, we directly deduce the following result.

Corollary 3.15. For any GPO game $(N, c) \in \Gamma$ such that $M \subset N$ and $Q(M)<V$, it holds that $N$ is externally stable.

Since big buyers are mutually exclusive and cannot coexist in a GPO in many cases (recall that we have $Q(M \cap N)<V$ in Propositions 3.11 and 3.14), it is worth to concentrate on GPOs comprising only one big buyer. The following result follows directly from Proposition 3.11.

Corollary 3.16. For any GPO game $(N \cup\{j\}, c) \in \Gamma$ such that $Q(N)<V$ and $j \in \mathcal{U} \backslash(N \cup M)$, the Shapley value is given by:

$$
S h_{i}(N \cup\{j\}, c)=\frac{1}{2} a_{m i n} q_{i}+\frac{1}{2} p(Q(N)) q_{i}+\frac{1}{6} b q_{i} Q(N \backslash\{i\}), i \in N,
$$

and

$$
S h_{j}(N \cup\{j\}, c)=a_{\text {min }} q_{j}-\frac{1}{2}\left(p(Q(N))-a_{m i n}\right) Q(N)-\frac{1}{3} b \sum_{t, m \in N} q_{t} q_{m} .
$$

\footnotetext{
${ }^{6} \mathrm{~A}$ cost TU-game $(N, c) \in \Gamma$ is additive if $c(S)=\sum_{i \in S} c(\{i\})$ for all $S \subseteq N$.
} 
It is worth noting that the cost $S h_{j}(N \cup\{j\}, c)$ big buyer $j$ has to pay is less than her individual cost $c(\{j\})=a_{\min } q_{j}$, as a consequence of the subadditive property of GPO games. Hence, big buyer $j$ has interest to join coalition $N$. Moreover, for any $i \in N$, since $Q(N)<V$, it follows from Proposition 3.1 that $S h_{i}(N, c)=p(Q(N)) q_{i}$. Hence, for any $i \in N$, we have:

$$
\begin{aligned}
S h_{i}(N \cup\{j\}, c)-S h_{i}(N, c) & =\frac{1}{2} a_{\min } q_{i}+\frac{1}{2} p(Q(N)) q_{i}+\frac{1}{6} b q_{i} Q(N \backslash\{i\})-p(Q(N)) q_{i} \\
& =\frac{1}{2} q_{i}\left(a_{\min }+\frac{1}{3} b Q(N \backslash\{i\})-(a-b Q(N))\right) .
\end{aligned}
$$

Therefore, for any $i \in N$, it holds that:

$$
S h_{i}(N \cup\{j\}, c)-S h_{i}(N, c)<0 \Longleftrightarrow a-b Q(N)-a_{\min }>\frac{1}{3} b Q(N \backslash\{i\}) .
$$

Thus, if the difference between the price $p(Q(N))$ and the best discount price $a_{\text {min }}$ is larger than or equal to the critical values $\frac{1}{3} b Q(N \backslash\{i\})$ for all $i \in N$, with a strict inequality for at least one member $i \in N$, then the small buyers will accept big buyer $j$ within the GPO. Hence, it follows directly from Corollary 3.16 the following result.

Corollary 3.17. For any GPO game $(N, c) \in \Gamma$ such that $N=M$, it holds that $N$ is externally stable if and only if there exists $i \in N$ such that $a-b Q(N)-a_{\min }<\frac{1}{3} b Q(N \backslash\{i\})$, or $a-$ $b Q(N)-a_{\min } \leq \frac{1}{3} b Q(N \backslash\{i\})$ for all $i \in N$.

To complete our analysis, we focus on the recruitment of small buyers within GPOs comprising only one big buyer. The following result is a consequence of Proposition 3.11.

Proposition 3.18. For any GPO game $(N, c) \in \Gamma$ such that $N=\{1,2, \ldots, n\}, Q(N \backslash\{n\})<V$ and $q_{n}>V$, if there exists $j \in M \backslash N$ then:

$$
S h_{i}(N \cup\{j\}, c)-S h_{i}(N, c)=-\frac{b q_{i} q_{j}}{3}<0, i \in N \backslash\{n\},
$$

and

$$
S h_{n}(N \cup\{j\}, c)-S h_{n}(N, c)=-\frac{1}{2} q_{j}\left(p(Q(N \cup\{j\} \backslash\{n\}))-a_{\min }-\frac{1}{3} b Q(N \backslash\{n\})\right) .
$$

Thus, if a small buyer $j$ joins a GPO comprising only one big buyer, the costs of all small buyers will decrease. Therefore, small buyer $j$ is welcome to join the GPO by small buyers in $N \backslash\{n\}$. However, big buyer $n$ will accept small buyer $j$ if and only if $p(Q(N \cup\{j\} \backslash\{n\}))-a_{\min }-$ $\frac{1}{3} b Q(N \backslash\{n\}) \geq 0$. Hence, we directly deduce the following result.

Corollary 3.19. For any GPO game $(N, c) \in \Gamma$ such that $N=\{1,2, \ldots, n\}, Q(N \backslash\{n\}<V$ and $q_{n}>V$, if there exists $j \in M \backslash N$ such that $p(Q(N \cup\{j\} \backslash\{n\}))-a_{\min }-\frac{1}{3} b Q(N \backslash\{n\}) \geq 0$, then $N$ is not externally stable. 


\section{Conclusion}

Different conceptions of stability of coalitions are envisaged in literature, and a contribution of our article is to propose a new one, based on the idea of exclusion (internal stability) or integration (external stability) of players regarding how they affect allocations within coalitions. Through the study of GPOs and the responses given with regard to cost allocations as well as their (un)-stability, we have mainly showed that small buyers have interest to cooperate within GPOs to benefit from the best price discount, which is not generally the case for big buyers who are mutually exclusive. Obviously, these results crucially depend on the preselected value (the Shapley value) to allocate costs within GPOs. We are convinced that the notions of internal and external stability will be very promising in the study of coalition formation in a wide variety of other situations such as, among many others, transportation, natural resources and cartels.

Lastly, it is important to observe that these notions can also be used for dealing with the stability of partitions ${ }^{7}$ instead of coalitions. A partition $P$ of a set of players $\mathcal{U}$ is stable with respect to $\phi$ if each coalition $N \in P$ is both internally and externally stable, with the new condition in external stability that the allocation of any joining player is evaluated when passing from a coalition in $P$ to another one. The study of stable partitions in allocation problems is left for future research.

\section{Appendix}

In this section, for notational convenience, let $\alpha_{s}=\frac{(s) !(n-s-1) !}{n !}$ for all $S \subseteq N$.

Proof of Proposition 3.1: It follows from (2.1) that:

\footnotetext{
${ }^{7} \mathrm{~A}$ family of sets $P$ is a partition of a set $\mathcal{U}$ if and and only if (i) $\emptyset \notin P$, (ii) $\bigcup_{N \in P} N=\mathcal{U}$, and (iii) for all $N_{1}, N_{2} \in P, N_{1} \neq N_{2} \Longrightarrow N_{1} \cap N_{2}=\emptyset$.
} 


$$
\begin{aligned}
S h_{i}(N, c) & =\sum_{S \subseteq N \backslash\{i\}} \alpha_{s}(C(S \cup\{i\})-C(S)) \\
& =\sum_{S \subseteq N \backslash\{i\}} \alpha_{s}\left(a q_{i}-b q_{i}^{2}-2 b q_{i} \sum_{k \in S} q_{k}\right) \\
& =a q_{i}-b q_{i}^{2}-2 b q_{i} \sum_{S \subseteq N \backslash\{i\}} \alpha_{s} \sum_{k \in S} q_{k} \\
& =a q_{i}-b q_{i}^{2}-2 b q_{i} \sum_{k \in N \backslash\{i\}} \sum_{S \subseteq N:} \alpha_{s} q_{k} \\
& =a q_{i}-b q_{i}^{2}-2 b q_{i} \sum_{k \in N \backslash\{i\}} q_{k} \sum_{s=1}^{n-1}\left(\begin{array}{l}
n-2 \\
s-1
\end{array}\right) \frac{s !(n-s-1) !}{n !} \\
& =a q_{i}-b q_{i}^{2}-2 b q_{i} \sum_{k \in N \backslash\{i\}} q_{k} \sum_{s=1}^{n-1} \frac{s}{n(n-1)} \\
& =a q_{i}-b q_{i}^{2}-2 b q_{i} \sum_{k \in N \backslash\{i\}} \frac{q_{k}}{2} \\
& =a q_{i}-b q_{i}^{2}-b q_{i} Q(N \backslash\{i\}) \\
& =q_{i}(a-b Q(N)), \quad
\end{aligned}
$$

which concludes the proof.

Proof of Proposition 3.4: First, we prove that the GPO game $(N, c) \in \Gamma$ can be decomposed into two cost TU-games, one of which is symmetric. ${ }^{8}$ To this end, we define two new cost TU-games $\left(N, c^{1}\right) \in \Gamma$ and $\left(N, c^{2}\right) \in \Gamma$ given by:

$$
c^{1}(S)=(a-b Q(S)) Q(S), S \subseteq N,
$$

and

$$
c^{2}(S)= \begin{cases}\left(a_{\min }-a+b Q(N)\right) Q(N), & S=N \\ 0, & S \subset N .\end{cases}
$$

Then, it holds that:

$$
\begin{aligned}
c(N) & =a_{\min } Q(N) \\
& =(a-b Q(N)) Q(N)+\left(a_{\min }-a+b Q(N)\right) Q(N) \\
& =c^{1}(N)+c^{2}(N),
\end{aligned}
$$

which implies that $c=c^{1}+c^{2}$. Since $\left(N, c^{2}\right)$ is a symmetric game, it is known that the Shapley value coincides with the equal division value, i.e., we have $S h_{i}\left(N, c^{2}\right)=\frac{\left(a_{\min }-a+b Q(N)\right) Q(N)}{n}$ for all $i \in N$. The additivity of the Shapley value together with Proposition 3.1 permits to conclude

\footnotetext{
${ }^{8} \mathrm{~A}$ cost TU-game is symmetric if $s=t \Longrightarrow c(S)=c(T)$ for all $S, T \subseteq N$.
} 
the proof.

Proof of Proposition 3.9: We want to prove that at least one small buyer in $N$ will reject the joining of any big buyer $j \in \mathcal{U} \backslash M$, i.e., there exists $k \in N$ such that $S h_{k}(N \cup\{j\}, c)>S h_{k}(N, c)$. First, note that for any $S \subseteq N$, we have $p(Q(S)) \geq a_{\text {min }}$ which implies that $p(Q(S)) Q(S) \geq$ $a_{\text {min }} Q(S)$. Therefore, it holds that:

$$
\begin{aligned}
c(S \cup\{j\})-c(S) & =a_{\min } Q(S \cup\{j\})-p(Q(S)) Q(S) \\
& \leq a_{\min } Q(S \cup\{j\})-a_{\min } Q(S) \\
& =a_{\min } q_{j} .
\end{aligned}
$$

By a similar argument, we have $c(S \cup\{j\})-c(S)<a_{\min } q_{j}$ for all $S \subset N$. Hence, it holds that:

$$
\begin{aligned}
S h_{j}(N \cup\{j\}, c) & =\sum_{\substack{S \subseteq N: \\
S \not \supset j}} \frac{s !(n-s) !}{(n+1) !}(C(S \cup\{j\})-C(S)) \\
& <\sum_{\substack{S \subseteq N: \\
S \ngtr j}} \frac{s !(n-s) !}{(n+1) !} a_{\min } q_{j} \\
& =a_{\min } q_{j},
\end{aligned}
$$

where the last equality holds because $\sum_{S \subseteq N: S \not \supset j} \frac{s !(n-s) !}{(n+1) !}=1$.

Second, since the Shapley value is efficient, we have:

$$
\begin{aligned}
\sum_{i \in N \cup\{j\}} S h_{i}(N \cup\{j\}, c) & =c(N \cup\{j\}) \\
& =a_{\min } Q(N \cup\{j\}) .
\end{aligned}
$$

Hence, it follows from (4.1) that:

$$
\begin{aligned}
\sum_{i \in N} S h_{i}(N \cup\{j\}, c) & =a_{\min } Q(N \cup\{j\})-S h_{j}(N \cup\{j\}, c) \\
& >a_{\min } Q(N) .
\end{aligned}
$$

Moreover, since $Q(N) \geq V$ we also have:

$$
\begin{aligned}
\sum_{i \in N} S h_{i}(N, c) & =c(N) \\
& =a_{\min } Q(N),
\end{aligned}
$$

where the first equality holds by the efficiency of the Shapley value. Thus, we conclude from (4.1) and (4.2) that there exists a buyer $k \in N$ such that: 


$$
S h_{k}(N \cup\{j\}, c)>S h_{k}(N, c),
$$

which concludes the proof.

Proof of Proposition 3.11: First, we want to prove (3.3). For any $i \in T$, it holds that:

$$
\begin{aligned}
S h_{i}(N, c) & =\sum_{\substack{S \subseteq N \backslash\{i\}\\
}} \alpha_{s}(c(S \cup\{i\})-c(S)) \\
& =\sum_{\substack{S \subseteq T: \\
S \ngtr i}} \alpha_{s}(c(S \cup\{i\})-c(S))+\sum_{\substack{S \nsubseteq T: \\
S \not 7 i}} \alpha_{s}(c(S \cup\{i\})-c(S)) \\
& =\sum_{\substack{S \subseteq T: \\
S \ngtr 7}} \alpha_{s}\left(a q_{i}-b q_{i}\left(2 Q(S)+q_{i}\right)\right)+\sum_{\substack{S \nsubseteq T: \\
S \ngtr 7}} \alpha_{s} a_{m i n} q_{i} \\
& =\sum_{\substack{S \subseteq T: \\
S \ngtr i}} \alpha_{s}\left(a q_{i}-b q_{i}^{2}\right)-\sum_{\substack{S \subseteq T: \\
S \ngtr i}} 2 \alpha_{s} b q_{i} Q(S)+\sum_{\substack{S \nsubseteq T: \\
S \ngtr 7}} \alpha_{s} a_{\min } q_{i} .
\end{aligned}
$$

Now, we want to study the three parts of equality (4.3). For the first part of equality (4.3), we have:

$$
\begin{aligned}
\sum_{\substack{S \subseteq T: \\
S \ngtr i}} \alpha_{s}\left(a q_{i}-b q_{i}^{2}\right) & =\sum_{s=0}^{t-1}\left(\begin{array}{c}
t-1 \\
s
\end{array}\right) \frac{s !(n-s-1) !}{n !}\left(a q_{i}-b q_{i}^{2}\right) \\
& =\sum_{s=0}^{t-1} \frac{(t-1) !(n-s-1) !}{n !(t-s-1) !}\left(a q_{i}-b q_{i}^{2}\right) \\
& =\frac{(t-1) !(n-t) !}{n !} \sum_{s=0}^{t-1}\left(\begin{array}{c}
n-s-1 \\
n-t
\end{array}\right)\left(a q_{i}-b q_{i}^{2}\right) \\
& =\frac{(t-1) !(n-t) !}{n !}\left(\begin{array}{c}
n \\
n-t+1
\end{array}\right)\left(a q_{i}-b q_{i}^{2}\right) \\
& =\frac{a q_{i}-b q_{i}^{2}}{n-t+1} .
\end{aligned}
$$

For the second part of equality (4.3), we have: 


$$
\begin{aligned}
\sum_{\substack{S \subseteq T: \\
S \not \ngtr i}} 2 \alpha_{s} b q_{i} Q(S) & =2 b q_{i} \sum_{k \in T \backslash\{i\}} q_{k} \sum_{\substack{S \subseteq T: \\
S \not \supset i, S \ni k}} \alpha_{s} \\
& =2 b q_{i} \sum_{k \in T \backslash\{i\}} q_{k} \sum_{s=1}^{t-1}\left(\begin{array}{c}
t-2 \\
s-1
\end{array}\right) \frac{s !(n-s-1) !}{n !} \\
& =2 b q_{i} \sum_{k \in T \backslash\{i\}} q_{k} \sum_{s=1}^{t-1} s\left(\begin{array}{c}
n-s-1 \\
n-t
\end{array}\right) \frac{(t-2) !(n-t) !}{n !} \\
& =2 b q_{i} \sum_{k \in T \backslash\{i\}} q_{k}\left(\begin{array}{c}
n \\
n-t+2
\end{array}\right) \frac{(t-2) !(n-t) !}{n !} \\
& =2 b q_{i} \sum_{k \in T \backslash\{i\}} \frac{q_{k}}{(n-t+1)(n-t+2)} .
\end{aligned}
$$

For the third part of equality (4.3), we have:

$$
\begin{aligned}
\sum_{\substack{S \nsubseteq T: \\
S \ngtr i}} \alpha_{s} a_{m i n} q_{i} & =a_{\min } q_{i}\left(\sum_{\substack{S \subseteq N \\
S \ngtr i}} \frac{s !(n-s-1) !}{n !}-\sum_{\substack{S \subseteq T: \\
S \ngtr i}} \frac{s !(n-s-1) !}{n !}\right) \\
& =a_{\min } q_{i}\left(1-\frac{1}{n-t+1}\right),
\end{aligned}
$$

where the last equality holds because $\sum_{S \subseteq T: S \not \supset i} \frac{s !(n-s-1) !}{n !}=\frac{1}{n-t+1}$. Therefore, for any $i \in T$, it follows from (4.4), (4.5) and (4.6) that:

$$
\begin{aligned}
S h_{i}(N, c) & =\frac{a q_{i}-b q_{i}^{2}}{n-t+1}-2 b q_{i} \sum_{k \in T \backslash\{i\}} \frac{q_{k}}{(n-t+1)(n-t+2)}+a_{m i n} q_{i}\left(1-\frac{1}{n-t+1}\right) \\
& =a_{m i n} q_{i} \frac{n-t}{n-t+1}+\frac{p(Q(T)) q_{i}}{n-t+1}+\frac{(n-t) b q_{i} Q(T \backslash\{i\})}{(n-t+1)(n-t+2)}
\end{aligned}
$$

Second, we want to prove (3.4). For any $i \in N \backslash T$, it holds that: 


$$
\begin{aligned}
& S h_{i}(N, c)=\sum_{S \subseteq N \backslash\{i\}} \alpha_{s}(c(S \cup\{i\})-c(S)) \\
& =\sum_{\substack{S \subseteq T: \\
S \not \supset i}} \alpha_{s}(c(S \cup\{i\})-c(S))+\sum_{\substack{S \nsubseteq T: \\
S \ngtr i}} \alpha_{s}(c(S \cup\{i\})-c(S)) \\
& =\sum_{\substack{S \subseteq T: \\
S \ngtr i}} \alpha_{s}\left(a_{\min } Q(S \cup\{i\})-(a-b Q(S)) Q(S)\right)+\sum_{\substack{S \complement T: \\
S \ngtr i}} \alpha_{s} a_{\min } q_{i} \\
& =\sum_{\substack{S \subseteq T: \\
S \not 7 i}} \alpha_{s}\left(a_{\min } Q(S)-(a-b Q(S)) Q(S)\right)+\sum_{\substack{S \subseteq T: \\
S \not \ngtr i}} \alpha_{s} a_{\min } q_{i}+\sum_{\substack{S \nsubseteq T: \\
S \not \ngtr i}} \alpha_{s} a_{\min } q_{i} \\
& =\sum_{\substack{S \subseteq T: \\
S \not \supset i}} \alpha_{s}\left(a_{\min } Q(S)-(a-b Q(S)) Q(S)\right)+a_{\min } q_{i} \\
& =\sum_{\substack{S \subseteq T: \\
S \not \supset i}} \alpha_{s}\left(a_{\min }-a\right) Q(S)+\sum_{\substack{S \subseteq T: \\
S \not \supset i}} \alpha_{s} b Q(S)^{2}+a_{\min } q_{i} .
\end{aligned}
$$

Now, we want to study the first two parts of equality (4.7). For the first part of equality (4.7), we have:

$$
\begin{aligned}
\sum_{\substack{S \subseteq T: \\
S \ngtr i}} \alpha_{s}\left(a_{\min }-a\right) Q(S) & =\left(a_{\min }-a\right) \sum_{\substack{S \subseteq T: \\
S \ngtr \ngtr i}} \alpha_{s} Q(S) \\
& =\left(a_{\min }-a\right) \sum_{k \in T} \sum_{\substack{S \subseteq T \\
S \ngtr \ngtr i, S \ni k}} \alpha_{s} q_{k} \\
& =\left(a_{\min }-a\right) Q(T) \sum_{s=1}^{t}\left(\begin{array}{c}
t-1 \\
s-1
\end{array}\right) \frac{s !(n-s-1) !}{n !} \\
& =\left(a_{\min }-a\right) \frac{(t-1) !(n-t-1) !}{n !} Q(T) \sum_{s=1}^{t} s\left(\begin{array}{c}
n-s-1 \\
n-t-1
\end{array}\right) \\
& =\left(a_{\min }-a\right) \frac{(t-1) !(n-t-1) !}{n !} Q(T) \sum_{s=1}^{n-r} s\left(\begin{array}{c}
n-s-1 \\
r-1
\end{array}\right) \\
& =\left(a_{\min }-a\right) \frac{(t-1) !(n-t-1) !}{n !} Q(T)\left(\begin{array}{c}
n \\
n-r+1
\end{array}\right) \\
& =\left(a_{\min }-a\right) \frac{Q(T)}{(n-t+1)(n-t)},
\end{aligned}
$$

where $r=n-t$ in the fifth equality. For the second part of equality (4.7), we have: 


$$
\begin{aligned}
& \sum_{\substack{S \subseteq T: \\
S \ngtr i}} \alpha_{s} b Q(S)^{2}=\sum_{\substack{S \subseteq T: \\
S \not 7 i}} \alpha_{s}\left(2 b \sum_{t, r \in S} q_{t} q_{r}+b \sum_{k \in S} q_{k}^{2}\right) \\
& =2 b \sum_{t, r \in T} q_{t} q_{r} \sum_{\substack{S \subseteq T: \\
S \ni t, r}} \alpha_{s}+\frac{b \sum_{k \in T} q_{k}^{2}}{(n-t+1)(n-t)} \\
& =2 b \sum_{t, r \in T} q_{t} q_{r} \sum_{s=2}^{t}\left(\begin{array}{l}
t-2 \\
s-2
\end{array}\right) \frac{s !(n-s-1) !}{n !}+\frac{b \sum_{k \in T} q_{k}^{2}}{(n-t+1)(n-t)} \\
& =2 b \sum_{t, r \in T} q_{t} q_{r} \frac{(t-2) !(n-t-1) !}{n !} \sum_{s=2}^{t} s(s-1)\left(\begin{array}{c}
n-s-1 \\
n-t-1
\end{array}\right) \\
& +\frac{b \sum_{k \in T} q_{k}^{2}}{(n-t+1)(n-t)} \\
& =2 b \sum_{t, r \in T} q_{t} q_{r} \frac{(t-2) !(n-t-1) !}{n !} 2\left(\begin{array}{c}
n \\
n-t+2
\end{array}\right)+\frac{b \sum_{k \in T} q_{k}^{2}}{(n-t+1)(n-t)} \\
& =\frac{4 b \sum_{t, r \in T} q_{t} q_{r}}{(n-t)(n-t+1)(n-t+2)}+\frac{b \sum_{k \in T} q_{k}^{2}}{(n-t+1)(n-t)} \text {, }
\end{aligned}
$$

where $\sum_{s=2}^{t} s(s-1)\left(\begin{array}{c}n-s-1 \\ n-t-1\end{array}\right)=2\left(\begin{array}{c}n \\ n-t+2\end{array}\right)$ from the fourth to the fifth equality can be proved by induction on $t$. Therefore, for any $i \in N \backslash T$, it follows from (4.8) and (4.9) that:

$$
\begin{aligned}
S h_{i}(N, c)= & a_{\text {min }} q_{i}+\frac{\left(a_{\text {min }}-a\right) Q(T)}{(n-t+1)(n-t)}+\frac{4 b \sum_{t, r \in T} q_{t} q_{r}}{(n-t)(n-t+1)(n-t+2)} \\
& +\frac{b \sum_{k \in T} q_{k}^{2}}{(n-t+1)(n-t)} \\
= & a_{\text {min }} q_{i}-\frac{a-b Q(T)-a_{\text {min }}}{(n-t+1)(n-t)} Q(T)-\frac{\sum_{t, r \in T} 2 b q_{t} q_{r}}{(n-t+1)(n-t+2)}
\end{aligned}
$$

which concludes the proof.

\section{References}

[1] Bloch, R.E., Perlman, S.P., Brown, J.S., 2008, An Analysis of Group Purchasing Organizations' Contracting Practices Under the Antitrust Laws: Myth and Reality. Mayer, Brown, Rowe and Maw Report.

[2] Chun, Y., 1991, On the symmetric and weighted Shapley values. International Journal of Game Theory, 20, 183-190.

[3] D’Aspremont, C., Gabszewicz, J.J., Jacquemin, A., Weymark, J.W., 1983, On the stability of collusive price leadership. Canadian Journal of Economics, 16, 17-25. 
[4] Driessen T., Funaki Y., 1991, Coincidence of and collinearity between game theoretic solutions. OR Spektrum, 13, 15-30.

[5] Doucette, W.R., 1997, Influences on member commitment to group purchasing organizations. Journal of Business Research, 40, 183-189.

[6] Hou, D., Sun, H., Sun, P., Driessen, T., 2018, A note on the Shapley value for airport cost pooling game. Games and Economic Behavior, 108, 162-169.

[7] Li, L., 2019, Cooperative purchasing and preactive inventory sharing - Channel balancing and performance improvement. European Journal of Operational Research, 278, 738-751.

[8] Moretti, S., Patrone, F., 2008, Transversality of the Shapley value. Top, 16, 1-41.

[9] Moulin, H., 1988, Axioms of cooperative decision making. Cambridge University Press.

[10] von Neumann, J., Morgenstern, O., 1944, Theory of Games and Economic Behavior. Princeton University Press.

[11] Safaei, A. S., Heidarpoor, F., Paydar, M. M., 2017, A novel mathematical model for group purchasing in healthcare. Operations Research for Health Care, 15, 82-90.

[12] Schotanus, F., 2007, Horizontal Cooperative Purchasing. Ph.D. Thesis, University of Twente, Netherlands.

[13] Schotanus, F., Telgen, J., De Boer, L., 2008, Unfair allocation of gains under the Equal Price allocation method in purchasing groups. European Journal of Operational Research, 187, 162-176.

[14] Shapley, L.S., 1953, A value for n-person games. Annals of Mathematics Study, 28, 307-317 (Princeton University Press).

[15] Shapley, L.S., 1955, Markets as cooperative games. RAND Corporation Paper P-629, 1-5.

[16] Thoron, S., 1998, Formation of a coalition-proof stable cartel. Canadian Journal of Economics, 31, 63-76.

[17] Yang, Y-C., Cheng, H. K., Ding, C., Li, S., 2017, To join or not to join group purchasing organization: A vendor's decision. European Journal of Operational Research, 258, 581589.

[18] Young, P., 1985, Monotonic solutions of cooperative games. International Journal of Game Theory, 14, 65-72.

[19] Zentes, J., Swoboda, B., 2000, Allied groups on the road to complex networks. Technology in Society, 22, 133-150. 